\title{
Domains with Strong Barrier
}

José L. Fernández

Dedicated to the Memory of J. L. Rubio de Francia

\section{Introduction}

The level sets of any Riemann mapping $f$ can not be arbitrarily long. More precisely, there exists an absolute constant $P$ so that if $\Omega$ is a plane simply connected domain, $f$ a Riemann mapping onto $\Omega$ and $L$ is an straight line then

$$
\text { length }\left(f^{-1}(\Omega \cap L)\right) \leqslant P .
$$

This beautiful result was first proved by Hayman and $\mathrm{Wu}[\mathrm{HW}]$, and a bit later by Garnett, Gehring and Jones, [GGJ]. See [FHM] for a simple proof, where it is shown that one can take $P=4 \pi^{2}$ and a conjecture as to the correct value of $P$ is offered.

One wonders as to what is the role of simple connectivity in the HaymanWu theorem. Let us call a domain $\Omega$ in the plane a Hayman-Wu domain if there exists a constant $C(\Omega)$ so that

$$
\text { length }\left(F^{-1}(\Omega \cap L)\right) \leqslant C(\Omega)
$$

for any straight line $L$ and universal cover $F$ from the unit disk $\Delta$ onto $\Omega$.

It was shown in $[\mathrm{FH}]$ that domains of finite connectivity with no complementary components consisting of a single point are Hayman-Wu domains. A word of caution: in $[\mathrm{FH}]$ one is not concerned with the dependence of the constant of $(0.1)$ upon $F$, but the argument applies. Moreover, it is easy to see that the punctured disk, $\Delta^{*}$, is not a Hayman-Wu domain, so that the non-degeneracy condition on the complementary components is essential. 
Let $\Gamma$ denote a covering group of the domain $\Omega$, i.e., a fix-point free discrete group of Möbius transformations of $\Delta$ with quotient $\Delta / \Gamma$ conformally equivalent to $\Omega$. Any two covering groups of $\Omega$ are conjugate, and conversely.

With $\Gamma$ we associate the invariant function

$$
U_{\Gamma, t}(z)=U_{t}(z)=\sum_{t \in \Gamma}\left(1-[z, T z]^{2}\right)^{t}
$$

where

$$
[a, b]=\left|\frac{a-b}{1-\bar{a} b}\right| .
$$

In $[\mathrm{FH}]$ it was shown (see Section 6 for the proof).

Theorem A. If $U_{1 / 2}$ is bounded in $\Delta$ then $\Omega$ is Hayman-Wu.

As a consequence of Theorem 2 one also has that if $\Omega$ is Hayman-Wu then $U_{1}$ is bounded. Notice that $U_{1} \leqslant U_{1 / 2}^{2}$.

The exponent of $\Gamma$ is defined as the exponent of convergence of the Dirichlet series

$$
\sum_{\gamma \in \Gamma} \exp (-s \rho(0, \gamma(0)))
$$

i.e. the smallest number $s$ which makes the series convergent. Here, and hereafter, $\rho(a, b)$ denotes the Poincaré distance between the points $a$ and $b$ on the unit disk; namely,

$$
\rho(a, b)=h([a, b]),
$$

with

$$
h(t)=\log \frac{1+t}{1-t}, \quad 0 \leqslant t \leqslant 1 .
$$

Since conjugate groups have the same exponent we may also speak of the exponent of $\Omega$. We shall use the notation $\delta(\Gamma), \delta(\Omega)$ to denote exponents.

It is an elementary fact that $\delta(\Omega) \leqslant 1$. Also, $\delta(\Omega) \geqslant 1 / 2$, if $\Gamma$ contains parabolic elements, or, equivalently if $\partial \Omega$ has isolated points.

Notice that the groups satisfying the hypothesis of Theorem A have exponent at most $1 / 2$.

Here we shall show the following somehow surprising result.

Theorem 1. If $\Omega$ is a Hayman-Wu domain then $\delta(\Omega)<1$. 
Since there are domains of finite connectivity (with no point-boundary components) with exponent arbitrarily close to 1 we see that Theorem 1 is in a certain sense sharp. The exponent of the domain

$$
\Omega_{\epsilon}=\Delta\left(0, \frac{1}{\epsilon}\right) \backslash \bar{\Delta}(0, \epsilon) \backslash \bar{\Delta}(1, \epsilon), \quad \epsilon \in\left(0, \frac{1}{2}\right)
$$

increases to 1 as $\epsilon$ decreases to 0 .

We shall deduce Theorem 1 from combining two results about domains with strong barriers.

Definition. Let $\Omega$ be a plane domain. A non-constant positive superharmonic function $U$ of $\Omega$ is called a strong barrier if there exists a positive number $\epsilon$ such that

$$
\Delta U+\frac{\epsilon \cdot U}{\operatorname{dist}(\cdot, \partial \Omega)^{2}} \leqslant 0
$$

(where this inequality is meant in the weak sense).

If $\Omega$ has a strong barrier then $\Omega$ has a Green's function and moreover every boundary point is regular for the Dirichlet problem, and thus $\Omega$ has no pointboundary components.

Domains with strong barriers can be characterized in a variety of ways, and we shall use the rich knowledge about them to prove the following two results which yield Theorem 1 immediately.

Theorem 2. If $\Omega$ is a Hayman-Wu domain then $\Omega$ posseses a strong barrier.

The reciprocal of Theorem 2 does not hold. This follows from Theorem 4 below.

Theorem 3. If $\Omega$ posseses a strong barrier then $\delta(\Omega)<1$.

In this case it is easy to see that the reciprocal does not hold; simply take $\Omega=\Delta^{*}$, then $\delta(\Omega)=1 / 2$, but $\Omega$ does not have a strong barrier.

It should be remarked that in [Po2] an example is offered of a domain with a strong barrier but $\delta=1$. There is an error in the calculations there.

A Denjoy domain is a domain in the sphere whose complement is a compact set of the real line. Thus $\Omega=\hat{\mathbb{C}} \backslash E, E \subset \mathbb{R}, E$ compact. Denjoy domains have been recently studied by several authors in connection mostly with the Corona problem. See [RR], [C], [JM], [GJ]. They provide a test case for problems about multiply connected domains. 
A compact set $E \subset \mathbb{R}$ is called homogeneous if there exists a constant $c_{E}$ so that if $x \in \mathbb{R}$ and $\delta>0$.

$$
\frac{|(x-\delta, x+\delta) \cap E|}{\delta} \geqslant c_{E} .
$$

Carleson introduced this condition in $[C]$ where he showed that the associated Denjoy domain satisfies the Corona theorem.

Garnett and Jones [GJ] later showed this with no restriction on the set $E$. More recently, Zinsmeister has shown that $E$ is homogeneous if and only if $H^{1}(E)=H^{1}(\mathbb{R})$ (see $[\mathrm{Z}]$ for definitions and results).

If $E$ is homogeneous then $\hat{\mathbb{C}} \backslash E$ has an strong barrier.

For Denjoy domains the homogeneity of the boundary is the key for being a Hayman-Wu domain.

Theorem 4. If $\Omega$ is a Denjoy domain, then $\Omega$ is a Hayman-Wu domain if and only if $\partial \Omega$ is homogeneous.

The proof of Theorem $i$ is in Section $i, i=2,3$, 4. In Section 5 we consider another notion of a domain being almost simply connected and relate that to the results above. In Section 6 we give the proof of Theorem A for the sake of completeness.

I wish to thank A. Ancona for pointing out the example in the Remark in Section 7. I am most grateful to Juha Heinonen for very stimulating conversations which motivated this paper.

\section{Domains with Strong Barrier}

Here we collect the relevant features of domains with strong barrier.

Let $\Omega$ be a plane domain other than the plane or a punctured plane. The universal covering Riemann surface is the unit disk. Consider the Poincaré metric in the unit disk. Via the universal covering map, $\pi$, it can be projected onto a metric in $\Omega$ so that $\pi$ is a local isometry. This projected metric is conformal with the euclidean metric and the scale factor, denoted by $\lambda_{\Omega}$, is determined by the equation

$$
\lambda_{\Omega}(\pi(z))\left|\pi^{\prime}(z)\right|=\frac{2}{1-|z|^{2}}, \quad z \in \Delta .
$$

The volume form of this metric will be denoted by $\omega_{\Omega}$; it is simply

$$
\omega_{\Omega}=\lambda_{\Omega}^{2} d x \wedge d y .
$$


It is always the case and follows from Schwarz's lemma that

$$
\lambda_{\Omega} \leqslant \frac{2}{\operatorname{dist}(\cdot, \partial \Omega)} .
$$

To have a reversed inequality, i.e., to have $0<\inf _{z \in \Omega} \lambda_{\Omega}(z) \operatorname{dist}(z, \partial \Omega)$ is equivalent to the existence of a strong barrier, [BP], [Po1]. Also in terms of the group $\Gamma$ we have that $\Omega$ has a strong barrier if and only if there exists $\tau_{0}>0$ so that the translation length of every element of $\Gamma$ is at least $\tau_{0}$, [P1]. (The translation length of a parabolic element is defined to be zero.) In geometric terms this translates into having no punctures plus the existence of a positive lower bound for the length of closed simple Poincaré geodesics of $\Omega$.

We shall need another characterization. A domain $\Omega$ has a strong barrier if and only if $\partial \Omega$ verifies the following capacity condition: there exists a constant $C_{0}>0$

$$
\operatorname{cap}(\Delta(b, r) \cap \partial \Omega)>C_{0} r
$$

for every $b \in \partial \Omega$, and $r, 0<r \leqslant \operatorname{diam}(\partial \Omega)$.

The strong barrier condition is also equivalent to $U_{\Gamma, 1}$ being bounded [Po2]. Recall that the condition appearing in Theorem $\mathrm{A}$ is that $U_{\Gamma, 1 / 2}$ is bounded.

All this can be found in [A], [BP], [Po1], [Po2].

\section{Proof of Theorem 2}

We will check that if $\Omega$ is a Hayman-Wu domain then $(a)$, there is a constant $\tau_{0}>0$ so that all closed simply geodesic have Poincaré length at least $\tau_{0}$, and (b), there are no punctures. We need a simple lemma:

Lemma. Let $T$ be hyperbolic Möbius transformation of the unit disk onto itself whose axis passes through 0 . Then

$$
\frac{1}{|T(0)|} \leqslant \sum_{k}\left(1-\left|T^{k}(0)\right|^{2}\right) \leqslant \frac{2}{|T(0)|^{2}} .
$$

Proof. We may assume that the fixed points of $T$ are -1 and 1 , and that $T(0)=a \in(0,1)$. Let $b_{n}=T^{n}(0), n \geqslant 0$. Then

$$
1-\left|b_{n}\right|^{2}=1-\left|T\left(b_{n-1}\right)\right|^{2}=\frac{\left(1-\left|b_{n-1}\right|^{2}\right)\left(1-|a|^{2}\right)}{\left|1+b_{n-1} a\right|^{2}}, \quad n \geqslant 1 .
$$

For $n \geqslant 1$ we have:

$$
1 \leqslant\left|1+b_{n-1} a\right| \leqslant 1+a,
$$


and so

$$
\left(1-a^{2}\right)^{n} \geqslant 1-\left|b_{n}\right|^{2} \geqslant\left(\frac{1-a}{1+a}\right)^{n}, \quad n \geqslant 1 .
$$

Therefore

$$
\frac{2}{a^{2}}>\sum_{k \in \mathbb{Z}}\left(1-\left|T^{k}(0)\right|^{2}\right)=1+2 \sum_{n=1}^{\infty}\left(1-\left|b_{n}\right|^{2}\right) \geqslant \frac{1}{a} .
$$

(a) Let $\sigma$ be a closed simple geodesic in $\Omega$.

The Jordan curve $\sigma$ contains points of $\partial \Omega$ in its Jordan interior. Let $s \in \sigma$ and $b \in \partial \Omega$ be such that

$$
|b-s|=\operatorname{dist}(\sigma, \partial \Omega \cap \text { interior }(\sigma)) .
$$

Let $F$ be a universal covering map which takes 0 to $s$.

Lift $\sigma$ to a geodesic segment in $\Delta$ through 0 . The lift is part of a diameter $\tilde{\sigma}$ of $\Delta$. Let $T$ be the Möbius covering transformation $(F \circ T=F)$ corresponding to $\sigma$. Then the axis of $T$ is $\tilde{\sigma}$ since $\sigma$ is smooth. Moreover the length $L$ of $\sigma$ safisfies

$$
\frac{1}{\tanh \left(\frac{L}{2}\right)} \leqslant \sum_{k \in \mathbb{Z}} 1-\left|T^{k}(0)\right|^{2} .
$$

The segment from $s$ to $b$ is contained in $\Omega$ and its preimage under $F$ contains a collection of curves each one of them emanates from a point of the orbit of 0 and goes all the way to $\partial \Delta$, therefore the total length of these curves is at least $\sum_{\gamma \in \Gamma} 1-|\gamma(0)|$. And consequently we have that

$$
\sum_{\gamma \in \Gamma}(1-|\gamma(0)|) \leqslant c_{\Omega} .
$$

Therefore,

$$
\tanh \left(\frac{L}{2}\right) \geqslant \frac{1}{2 c_{\Omega}} .
$$

and so $L$ is bounded below by a constant depending only on $c_{\Omega}$.

(b) It remains to deal with the possible isolated points of the boundary of $\Omega$. We may assume that $0 \in \partial \Omega$ and $\Delta^{*} \subset \Omega$. Let $F$ be a universal covering map which takes 0 to $1 / 2$. The circle $|z|=1 / 2$ is lifted to a curve joining 0 to $T(0)$ where $T \in \Gamma$ is parabolic. We may assume that the unique fixed point of $T$ is 1 . Now the segment $\sigma$ from $1 / 2$ to 0 lifts to a curve $\tilde{\sigma}$ in 
$\Delta$ which joins 0 to 1 . Notice that $F\left(\bigcup_{k \in \mathbb{Z}} T^{k}(\tilde{\sigma})\right) \subset(0,1 / 2]$, and therefore since $T(\tilde{\sigma})$ joins $\gamma(0)$ to 1 , we see that

$$
\sum_{k \in \mathbb{Z}}\left|1-T^{k}(0)\right| \leqslant \text { length }\left(F^{-1}(\Omega \cap \mathbb{R})\right) \leqslant c(\Omega) .
$$

But it is easy to see that $\left|1-T^{k}(0)\right||k| \rightarrow t_{0}$ as $|k| \rightarrow \infty$ where $t_{0}$ is a positive number. Therefore the sum on the left is actually infinite. Thus we have shown that $\partial \Omega$ has no isolated points and so the proof is complete.

\section{Proof of Theorem 3}

Our proof of Theorem 3 is actually a combination of results which appear in papers by Ancona [A] and Sullivan [S1]. Ancona shows that in domains with strong barrier the following form of Hardy's inequality holds: there exists a constant $c_{1}$ so that for every smooth function $\varphi$ compactly supported in $\Omega$

$$
\iint_{\Omega}|\varphi(z)|^{2} \frac{d x d y}{\operatorname{dist}(z, \partial \Omega)^{2}} \leqslant c_{1} \iint_{\Omega}|\nabla \varphi(z)|^{2} d x d y, \quad(z=x+i y) .
$$

The constant $c_{1}$ depends only on the $\epsilon$ in the definition of strong barrier. As a matter of fact the existence of strong barrier is equivalent to (3.1).

Recall that the density of the Poincare metric is denoted by $\lambda_{\Omega}$, while its volume form is denoted by $\omega_{\Omega}$.

The Dirichlet integral is a conformal invariant. Therefore the integral on the right hand side of the inequality (3.1) equals

$$
\iint_{\Omega}\left|\nabla_{\Omega} \varphi\right|_{\Omega}^{2} \omega_{\Omega}
$$

where $\nabla_{\Omega}$ denotes the gradient with respect to the Poincaré metric of $\Omega$, and $\mid I_{\Omega}$ denotes length in the tangent space with respect to the Poincaré metric of $\Omega$.

Moreover, it is always the case that

$$
\lambda_{\Omega}(z) \leqslant \frac{2}{\operatorname{dist}(z, \partial \Omega)}, \quad \text { for every } \quad z \in \Omega .
$$

Using (3.2) and (3.3) we see that inequality (3.1) implies that

$$
\iint_{\Omega}|\varphi|^{2} \omega_{\Omega} \leqslant c_{1} \iint_{\Omega}\left|\nabla_{\Omega} \varphi\right|^{2} \omega_{\Omega}, \text { for every } \varphi \in C_{0}^{\infty}(\Omega) .
$$

But this means that the Poincaré inequality holds in the Riemannian manifold $\Omega$ and therefore the spectrum of the Laplace-Beltrami operator of $\Omega$ is contained in $\left(-\infty,-1 / C_{1}\right)$. 
And now the theorem of Elstrodt-Patterson-Sullivan (see [S1, p. 333]) provides the final stroke because if $\delta=\delta(\Gamma)$ then it claims in our case that

$$
\delta(1-\delta) \geqslant \frac{1}{C_{1}},
$$

if $\delta \geqslant 1 / 2$. In particular,

$$
\delta \leqslant \max \left\{1-\frac{1}{C_{1}}, \frac{1}{2}\right\}<1 .
$$

Remark. One can use the argument of Lemma 1 of [Su] to show directly that if a domain posseses strong barrier then the isoperimetric inequality, $A<c L$, holds (for its Poincaré metric), and combine this with Cheeger's inequality to give the result.

\section{Proof of Theorem 4}

\section{Sufficiency}

Here we assume that $\partial \Omega$ is homogeneous.

First of all we reduce the proof to the case $L=\mathbb{R}$. Let a universal covering map $F$ be given and assume that we have seen that

$$
\text { length }\left(F^{-1}(\Omega \cap \mathbb{R})\right) \leqslant M
$$

where $M$ depends on $\Omega$ but not on $F$. Let $L$ be any other straight line and $L^{+}$ be the part of $L$ above $\mathbb{R}$. Let $G$ be any branch of $F^{-1}$ defined on the upper half plane. By the Hayman-Wu theorem (see [GGJ]) we have that

$$
\text { length }\left(G\left(L^{+}\right)\right) \leqslant \tilde{P} \text { length }(\partial G(U))
$$

where $\tilde{P}$ is an absolute constant. Adding up (4.2) over all branches $G$ and using (4.1) we see that

$$
\text { length }\left(F^{-1}\left(L^{+}\right)\right) \leqslant \tilde{M} \text {, }
$$

where $\tilde{M}$ depends only on $\Omega$. Similarly, length $\left(F^{-1}\left(L^{-}\right)\right) \leqslant \tilde{M}$ and so

$$
\text { length }\left(F^{-1}(L)\right) \leqslant 2 \tilde{M}
$$

Choose now a universal covering map $F$. We will check that (4.1) holds. Let us denote by $I_{j}$ the complementary intervals of $E$ in $\mathbb{R}$.

In each $I_{j}$ we select points $z_{k}^{(j)}$ as follows: if $I_{j}=(a, b)$, with $a, b$ finite then 


$$
z_{0}^{(j)}=\frac{a+b}{2}
$$

and

$$
z_{k}^{(j)}=z_{0}^{(j)}+\operatorname{sign}(k) \frac{\left|I_{j}\right|}{2}\left(1-1 / 2^{k}\right), \quad k \in \mathbb{Z} .
$$

If the interval contains $\infty$, we select $\infty$ as $z_{0}^{(j)}$ and, if $q=\sup E$ and $p=\inf E$,

we let

$$
\begin{array}{ll}
z_{k}^{(j)}=q+\frac{1}{2^{k}} \operatorname{diam}(E), & k \geqslant 1, \\
z_{k}^{(j)}=p-2^{k} \operatorname{diam}(E), & k \leqslant-1 .
\end{array}
$$

Let $Z=\left\{z_{k}^{(j)}: j, k\right\}$. We shall check that $F^{-1}(Z)$ is an interpolating sequence whose constants are independent of the choice of the universal covering $F$ of $\Omega$. Assume this for the moment and let us show how to finish the proof.

Denote by $I_{j, k}$ the interval $\left(z_{k}^{(j)}, z_{k+1}^{(j)}\right), k \in \mathbb{Z}$. Let $G$ be any branch of $F^{-1}$ defined on the whole interval $3 I_{j, k}$ (which is the interval with same center and triple the length). Then $G\left(I_{j, k}\right)$ is a curve in $\Delta$ whose Poincaré diameter is bounded by an absolute constant ( $\log 4)$; this follows from Schwarz' Lemma. In particular if $x \in I_{j, k}$ we have

$$
1-|G(x)|^{2} \leqslant A\left(1-\left|G\left(z_{k}^{(j)}\right)\right|^{2}\right),
$$

where $A$ is an absolute constant. Thus, if $x \in I_{j, k}$,

$$
\left|G^{\prime}(x)\right|=\left(1-|G(x)|^{2}\right) \lambda_{\Omega}(x) \leqslant \frac{1-|G(x)|^{2}}{\operatorname{dist}(x, \partial \Omega)} \leqslant \frac{1-|G(x)|^{2}}{\left|I_{j, k}\right|} \leqslant A \frac{1-\left|G\left(z_{k}^{(j)}\right)\right|^{2}}{\left|I_{j, k}\right|} .
$$

Consequently,

$$
\int_{I_{j, k}}\left|G^{\prime}(x)\right| d x \leqslant A\left(1-\left|G\left(z_{k}^{(j)}\right)\right|^{2}\right) .
$$

And, in particular, adding up (4.4) over all $j, k$ and $G$ we obtain that

$$
\text { length }\left(F^{-1}(\Omega \cap \mathbb{R})\right) \leqslant A \sum_{w \in F^{-1}(Z)}\left(1-|w|^{2}\right) \text {. }
$$

But we are assuming that we have already shown that $F^{-1}(Z)$ is an interpolating sequence and so, in particular, that the measure

$$
\mu=\sum_{w \in F^{-1}(Z)}\left(1-|w|^{2}\right) \delta_{w}
$$


is finite (as a matter of fact, that $\mu$ is a Carleson measure). The interpolation constants of $F^{-1}(Z)$ depend only on $\Omega$ and thus so does the mass of $\mu$; this implies that (4.1) holds.

All that remains is to show that $F^{-1}(Z)$ is an interpolating sequence. But before doing so let us remark that the argument above (which appears in [GGJ]) is general. In fact, given $\Omega$ (not necessarily Denjoy), split the intersection with $\Omega$ of a given line $L$ into disjoint intervals $J_{k}$ so that in each interval $J_{k}$

$$
\frac{1}{100} \leqslant \frac{\operatorname{dist}(z, \partial \Omega)}{\text { length }(J)} \leqslant 100 .
$$

Let $z_{k}$ be the center of $J_{k}$. Then if $F^{-1}\left(\left\{z_{k}\right\}\right)$ is interpolating with contants depending on $\Omega$ alone one deduces that $\Omega$ is a Hayman-Wu domain. Conversely, if $\Omega$ is Hayman-Wu then using that $\Omega$ has strong barrier one may show that the inverse image of such a sequence is interpolating.

There is an argument introduced by Garnett-Gehring-Jones for checking whether $F^{-1}(Z)$ is interpolating or not by transfering the problem to a harmonic measure estimate on $\Omega$ itself. If we assume that $\Omega$ has an strong barrier then we have that $F^{-1}(Z)$ is interpolating if and only if there is $\epsilon<1 / 4$ and $a>0$ so that if for $z \in Z$ we define

$$
H_{\epsilon}(z)=\sum_{z^{\prime} \in Z \backslash\{z\}} \bar{\Delta}\left(z^{\prime}, \epsilon \operatorname{dist}\left(z^{\prime}, \partial \Omega\right)\right) \cap \mathbb{R} .
$$

Then

$$
\omega\left(z, \partial \Omega, \Omega \backslash H_{\epsilon}(z)\right) \geqslant a, \text { for all } z \in Z .
$$

This appears in [Po2] and in [JM]. If $z^{\prime}=\infty$ by $\bar{\Delta}(\infty, \epsilon$ dist $(\infty, \partial \Omega))$ we mean $\overline{\mathbb{R}} \backslash(p-(1 / \epsilon) \operatorname{diam} \partial \Omega, q+(1 / \epsilon)$ diam $\partial \Omega)$. It turns out that for Denjoy domains with homogeneous complement (4.5) can be easily checked. This could be done as follows: if $z \in Z \backslash\{\infty\}$, then $\Delta(z,(1 / 8)$ dist $(z, \partial \Omega)) \subset \Omega \backslash H_{\epsilon}(z)$; by Harnack's inequality it is enough to estimate

$$
\omega\left(z+i d, \partial \Omega, \Omega \backslash H_{\epsilon}(z)\right)
$$

from below, where

$$
d=\frac{1}{16} \operatorname{dist}(z, \partial \Omega)
$$

But

$$
\omega\left(z+i d, \partial \Omega, \Omega \backslash H_{\epsilon}(z)\right) \geqslant \omega(z+i d, \partial \Omega, U),
$$

(where $U$ is the upper half plane). 
Let $b \in \partial \Omega$ be such that $|z-b|=\operatorname{dist}(z, \partial \Omega)$, using again Harnack's inequality we see that we just need to estimate $\omega(b+i d, E, U)$ form below. But from the explicit expression of the Poisson kernel of the upper half plane we readily see that

$$
\omega(b+i d, E, U) \geqslant C \frac{|(b-10 d, b+10 d)|}{d}
$$

where $C$ is an absolute constant. And this gives the desired result. (For $z=\infty$ one needs a minor variation of the argument.)

\section{Necessity}

Assume that $\Omega$ is a Hayman-Wu domain. We want to check that $\partial \Omega$ is homogeneous. Write $E=\partial \Omega$.

We already know that $E$ satisfies the capacity condition (1.1).

We use the notation of the proof of the sufficiency.

We know that for some $\epsilon>0$ and $a=a(\epsilon)>0$

$$
\omega\left(z, E, \Omega \backslash H_{\epsilon}(z)\right) \geqslant a, \text { for every } z \in A .
$$

It is easy to check that $E \cup \hat{H}_{\epsilon}(z)$ is homogeneous with a constant depending only on $\epsilon$ (and not on $E$ ). Here $\hat{H}_{\epsilon}(z)$ is the part of $H(z)$ not lying in the component of $\infty$ of $\bar{R} \backslash E$. Clearly

$$
\omega\left(z, E, \Omega \backslash \hat{H}_{\epsilon}(z)\right) \geqslant a, \quad \text { for every } \quad z \in Z .
$$

Let $V=[p, q]$ be the smallest interval containing $E$. We shall check that for an appropriate constant $M=M(\epsilon)$ we have for all $y \in V \backslash E$ that

$$
|\Delta(y, M \operatorname{dist}(y, E)) \cap E| \geqslant C \operatorname{dist}(y, E)
$$

where $C=C(\epsilon)$.

This will be enough as the following simple lemma shows.

Lemma. Let $A \subset[0,1]$ be a closed set and assume that there exist constants $\eta, N$ such that if $y \in[0,1] \backslash A$

$$
|(y-N d(y), y+N d(y)) \cap A| \geqslant \eta d(y),
$$

where $d(y)=\operatorname{dist}(y, A)$ then

$$
|A| \geqslant \eta / 8 N
$$

Proof of lemma. Let $J_{y}=(y-N d(y), y+N d(y))$. 
Consider

$$
B=\bigcup_{y \in[0,1] \backslash A} J_{y} .
$$

We may choose points $y_{j}$ so that

$$
B=\bigcup_{j} J_{y_{j}}
$$

and

$$
\sum_{j} \chi_{y_{j}} \leqslant 2 \chi_{I}
$$

(i.e. no point of $B$ is in more than two of the $J_{y_{j}}$ ). Then

$$
|A \cap B|=\int_{A} \chi_{B} \geqslant \frac{1}{2} \sum_{j}\left|A \cap J_{y_{j}}\right| \geqslant \frac{\eta}{2} \sum_{j} d\left(y_{j}\right) \geqslant \frac{\eta}{4 N}|B| .
$$

Now, $A \cap B \subset A$, and $B \supset[0,1] \backslash A$ so that

$$
|A| \geqslant \frac{\eta}{4 N}(1-|A|)
$$

and so

$$
|A| \geqslant \frac{\eta}{8 N}
$$

It is clear that in order to check (4.7) for all $y \in V \backslash E$ it is enough to do so when $y$ is one of the points $z_{k}^{(j)}$.

Since both the data and the desired conclusion are translation and scale invariant, we may assume that $z_{k}^{(j)}=0,1 \in E$, and $\operatorname{dist}\left(z_{k}^{(j)}, E\right)=1$.

Around $1 / 2$ there is an interval of length $2 \epsilon$ which lies in $\partial H_{\epsilon}(0)$. Then there exists $M=M(\epsilon)$ so that

$$
\omega\left(0, \mathbb{R} \backslash(-M(\epsilon), M(\epsilon)), \Omega \backslash \hat{H}_{\epsilon}(z) \backslash[-M(\epsilon), M(\epsilon)]\right) \leqslant a / 2 .
$$

Therefore we see that

$$
\omega\left(0, E \cap[-M(\epsilon), M(\epsilon)], \Omega \backslash \hat{H}_{\epsilon}(z)\right) \geqslant a / 2 .
$$

We define two sets $\tilde{E}, \tilde{K}$ as follows: we let $\tilde{E}$ be the set $E \cap[-M(\epsilon), M(\epsilon)]$ and $\tilde{K}$ be the set $E \cup\left([-M(\epsilon), M(\epsilon)] \cap \hat{H}_{\epsilon}(0)\right)$. Consider $\tilde{\Omega}=\hat{\mathbb{C}} \backslash \tilde{K}$. We know from (4.8) that

$$
\omega(0, \tilde{E}, \tilde{\Omega}) \geqslant a / 2 .
$$


Again $\tilde{K}$ is homogeneous with a constant depending only on $\epsilon$, and since $\tilde{K} \subset[-M(\epsilon), M(\epsilon)]$ then we know that $\omega(\infty, \bullet, \tilde{\Omega})$ is absolutely continuous with respect to length and in fact, that the Radon-Nikodym derivative $h$ is in $L^{p}$, for some $p>1$. More precisely.

$$
\omega(\infty, \cdot, \tilde{\Omega})=h d x
$$

and for $p=p(\epsilon)>1$ and $T=T(\epsilon)$ we have

$$
\int_{\partial \tilde{\Omega}}|h(x)|^{p} d x \leqslant T(\epsilon) .
$$

This is the heart of the matter. It is due to Jones and Marshall ([JM]).

From Harnack's inequality (and a bit of Poincaré geometry), we have

$$
\omega(\infty, \tilde{E}, \tilde{\Omega}) \geqslant a^{\prime} .
$$

$\left(a^{\prime}=a^{\prime}(a, \epsilon)=a^{\prime}(\epsilon)\right)$. Therefore

$$
a^{\prime} \leqslant \int_{E}|h(x)| d x \leqslant T(\epsilon)^{1 / p}|\tilde{E}|^{1-1 / p} .
$$

And so

$$
|E \cap[-M(\epsilon), M(\epsilon)]| \geqslant c=c(\epsilon)
$$

and we are done.

\section{Fully Accessible Domains}

This is a notion that has been introduced and studied by Patterson, [Pa1], [Pa2], Pommerenke [Po3], [Po4], [Po5], and Sullivan [S2]. A Fuchsian group $\Gamma$ is called fully accessible if the action of $\Gamma$ on $\partial \Delta$ is fully dissipative i.e. if there is a measurable set $B \subset \partial \Delta$ so that if $\gamma \in \Gamma \backslash\{i d\},|\gamma(B) \cap B|=0$ and $\left|\partial \Delta \backslash \bigcup_{\gamma \in \Gamma} \gamma(B)\right|=0$, or in other terms that the action of $\Gamma$ on $\partial \Delta$ has a measurable fundamental set.

A domain is called fully accessible if its covering group is fully accessible.

Patterson showed in [Pa1] that if $\delta(\Gamma)<1 / 2$ then $\Omega$ is fully accesible. On the other hand fully accesible domains may have $\delta(\Omega)=1$. One such example is provided by $\Omega=\Delta^{*} \backslash\left\{a_{n}\right\}$, where $a_{n} \rightarrow 0$. It is easy to see that $\Omega$ is fully accessible (see Theorem 3 or Example 1 in [Po4]) but $\delta(\Omega)=1$. See Remark 1 .

It is reasonable to expect that Hayman-Wu domains must be fully accesible. We can only show this for Denjoy domains. In that case a Hayman-Wu domain satisfies that if $F$ is the symmetric universal covering map with $F(0)=\infty, \Gamma$ its covering group, and $D_{0}$ the associated Dirichlet region at 0 then 


$$
\sum_{\gamma \in \Gamma} \text { length }\left(\partial\left(\gamma\left(D_{0}\right)\right)\right)<\infty
$$

(see $[\mathrm{FH}])$.

This clearly implies that

$$
\left|\partial \Delta \backslash \bigcup_{\gamma} \gamma\left(\partial D_{0} \cap \partial \Delta\right)\right|=0,
$$

which gives that $\Omega$ is fully accessible. Another argument to show this is provided by two characterizations. Assume that $\Omega$ is a Denjoy domain. We have seen that $\Omega$ is Hayman-Wu if and only if $\Omega$ is homogeneous; on the other hand it has been shown by D. Hamilton and the author that $\Omega$ is fully-accesible if and only if harmonic measure in $\partial \Omega$ is absolutely continuous with respect to arc length (see Remark 2). But Carleson, [C], showed that for homogeneous sets harmonic measure is in fact absolutely continuous.

Remark 1. Let $a_{n}$ be a sequence of numbers converging to zero. Let

$$
\Omega=\Delta^{*} \backslash\left\{a_{k}\right\}_{k=1}^{\infty} .
$$

Now

$$
\delta(\Omega) \geqslant \delta\left(\Delta \backslash\left\{0, a_{n}\right\}\right) .
$$

This follows from the results about signatures in [Pa2], but in $[\mathrm{F}]$ it is shown that $\delta\left(\Delta \backslash\left\{0, a_{n}\right\}\right) \rightarrow 1$ as $n \rightarrow \infty$ therefore $\delta(\Omega)=1$.

Remark 2. We simply sketch the argument. It is based on the special form of the Dirichlet's, $D_{0}$, and Green's, $G_{0}$, fundamental region associated to the covering map $F$ with takes 0 to $\infty$ and is symmetric under complex conjugation. The Dirichlet region is mapped under $F$ on $\mathbb{C} \backslash[p, q]$ where $[p, q]$ is the smallest closed interval which contains $\partial \Omega$. Since $\partial D_{0}$ is rectifiable it follows that if $\Gamma$ is fully accessible then $\omega(\infty, \bullet, \partial \Omega)$ is absolutely continuous with respect to length. Conversely, since the Green's region is mapped onto $\mathbb{C} \backslash[p, q]$ one sees that if $\omega(\infty, \cdot, \partial \Omega)$ is absolutely continuous with respect to length then the Green's measure is absolutely continuous with respect to $d \theta$, and this is equivalent to full accessibility; (see [Po3] for definitions and this last result).

\section{Proof of Theorem A}

We start with 
Lemma. Let $G$ be a Fuchsian group and denote by $D_{0}(G)$ the Dirichlet region of $G$ at 0 . Then

$$
\sum_{g \in G} \text { length }\left(\partial g\left(D_{0}(G)\right) \leqslant \pi^{2} \sum_{g \in G}\left(1-|g(0)|^{2}\right)^{1 / 2} .\right.
$$

Proof. The domain $g\left(D_{0}(G)\right)$ is contained in

$$
\{z: \rho(z, g(0)) \leqslant \rho(z, 0)\}=H(g(0)) .
$$

By a result of $\mathrm{B}$. Brown, $[\mathrm{B}]$, we have that

$$
\text { length }\left(\partial g\left(D_{0}(G)\right)\right) \leqslant \frac{\pi^{2}}{2} \operatorname{diam}\left(g\left(D_{0}(G)\right)\right) \text {. }
$$

But

$$
\operatorname{diam}(H(g(0)))=2\left(1-|g(0)|^{2}\right)^{1 / 2},
$$

and so the result follows.

If $\Gamma$ satisfies that $U_{1 / 2}$ is bounded then for any group $G$ conjugate to $\Gamma$ we have

$$
\sum_{g \in G} \text { length }\left(g\left(\partial D_{0}(G)\right)\right) \leqslant \pi^{2}\left\|U_{1 / 2}\right\|_{\infty}
$$

For $G=\omega^{-1} \Gamma \omega$, where $\omega \in \operatorname{Möb}(\Delta)$, and then

$$
\sum_{g \in G}\left(1-|g(0)|^{2}\right)^{1 / 2}=\sum_{\gamma \in G}\left(1-[\omega(0), \gamma(\omega(0))]^{2}\right)^{1 / 2}=U_{1 / 2}(\omega(0))
$$

Assume that a covering group $\Gamma$ of $\Omega$ (and hence all) has $\left\|U_{1 / 2}\right\|_{\infty}<\infty$.

Let $F$ be any universal covering map from $\Delta$ onto $\Omega$. The group of deck transformations of $\Gamma$ is a group $G$ conjugate to $\Gamma$.

We want to estimate the length of the set $V=F^{-1}(\Omega \cap L)$ where $L$ is an straight line. Since $F$ is one-to-one on $g\left(D_{0}(G)\right)$ we deduce form the Hayman$\mathrm{Wu}$ theorem (see [GGJ]) that

$$
\text { length }\left(V \cap g\left(D_{0}(G)\right)\right) \leqslant C \text { length }\left(g\left(\partial D_{0}(G)\right)\right)
$$

where $C$ is an absolute constant. But then using (6.1) and (6.2) we deduce that

$$
\begin{aligned}
\text { length }(V) & \leqslant \sum_{g \in G} \text { length }\left(\partial g\left(D_{0}(G)\right)\right)+\sum_{g \in G} \text { length }\left(V \cap g\left(D_{0}(G)\right)\right) \\
& \leqslant(1+c) \sum_{g \in G} \text { length }\left(g\left(\partial D_{0}(G)\right)\right) \\
& \leqslant(1+c) \pi^{2}\left\|U_{1 / 2}\right\|_{\infty} .
\end{aligned}
$$




\section{An Example}

We know that for a domain $\Omega, U_{1}$ is bounded if and only if $\Omega$ posseses a strong barrier. Possesing a strong barrier means that $\Omega$ contains no doubly connected domains (separating $\partial \Omega$ ) of arbitrarily large modulus, or equivalently, in view of a theorem of Teichmüller ([Ah, p. 74]), that contains no ring (separating $\partial \Omega$ ) of arbitrarily large modulus (see [BP], [Po1]).

Let us define the modulus of a domain $\Omega$ as

$$
M(\Omega)=\sup \{\bmod (R): R \text {, ring }, R \subset \Omega, R \text { separating } \partial \Omega\} .
$$

The constant $M(\Omega)$ and the reciprocal of the $\epsilon$ in the definition of strong barrier are bounded by functions of each other.

Since $\delta(\Omega)<1 / 2$ guarantees that there are no isolated boundary points it is tempting to guess that $\delta(\Omega)<1 / 2$ implies that $\Omega$ posseses an strong barrier. Theorem A also points in that direction. Unfortunately

Example. Given $\delta_{0}>0$ there exist a domain $\Omega$ with $\delta(\Omega) \leqslant \delta_{0}$ but $M(\Omega)=\infty$.

In order to show that the exponent of a domain is close to 1 one only has to provide an example of a function $\varphi \in C_{0}^{\infty}(\Omega)$ with small

$$
\frac{\iint_{\Omega}|\nabla \varphi|_{\Omega}^{2} d x d y}{\iint_{\Omega}|\varphi|^{2} \omega_{\Omega}} .
$$

But the Rayleigh quotient is of no help here since at most it can be used to show that $\delta(\Omega) \leqslant 1 / 2$. We do have to look into the geometry of $\Omega$.

Given a sequence $\epsilon_{j}$ of positive numbers tending to zero consider the domain

$$
\Omega=\mathbb{C} \backslash \bigcup_{n \in \mathbb{Z}} \bar{\Delta}\left(2 n+1, \epsilon_{|n|}\right) \backslash \bigcup_{n \in \mathbb{Z}} T\left(2 n, \eta_{n}\right) .
$$

where if $a \in \mathbb{R}$ and $\eta>0$

$$
T(a, \eta)=\{a+i y:|y| \geqslant \eta\} \cup\{x+i y:|x-a| \leqslant 1 / 2,|y|=\eta\} .
$$

If $\delta_{0}$ is given we can choose the numbers $\eta_{n}$ converging to zero so fast that $\delta(\Omega) \leqslant \delta_{0}$. Of course, $M(\Omega)=\infty$.

We content ourselves with giving a proof of the following

Lemma. Given $\delta_{0}>0$ and $M_{0}$ there exists a triply connected domain $\Omega$ with

$$
\delta(\Omega) \leqslant \delta_{0} \quad \text { and } \quad M(\Omega) \geqslant M_{0} .
$$


Consider the domain

$$
\Omega=\mathbb{C} \backslash \bar{\Delta}(1, \epsilon) \backslash \bar{\Delta}(-1, \epsilon) \backslash T(0, \eta) .
$$

The set $\{x+i y:|y| \leqslant \eta / 2,|x| \leqslant 1 / 2\}$ will be called the tunnel. It is clear that if $\epsilon$ is small enough then $M(\Omega) \geqslant M_{0}$ (recall that $\eta \leqslant \epsilon$ ). We now fix $\epsilon$ and show that $\delta(\Omega)$ tends to zero as $\eta \rightarrow 0$.

Notice that $\Omega$ is symmetric under reflection on the imaginary axis $\left((x+i y)^{*}=-x+i y\right)$. Choose $F$ so that $F(0)=1$ and $F(\bar{z})=F(z)^{*}$. We have to check that for $s$ small (assuming $\eta$ small) we have

$$
\sum_{\gamma \in \Gamma} e^{-s \rho(0, \gamma(0))}<\infty
$$

where $\Gamma$ is the covering group of $F$. The group $\Gamma$ is a free group in two generators. One generator, $\alpha$, corresponds to the loop with base at 1 , which surrounds $\bar{\Delta}(1, \epsilon)$ the other one, $\beta$, corresponds to the $*$-symmetric loop. We decompose the sum in (7.1) as follows

$$
1+\sum_{k=1}^{\infty} \sum_{\gamma \in A_{k}} e^{-s \rho(0, \gamma(0))},
$$

where $A_{k}$ denotes the collection of those elements of $\Gamma$ of the form

$$
\sigma=w_{1}^{p_{1}} w_{2}^{p_{2}} \cdots w_{k}^{p_{k}}
$$

where $w_{i}$ is $\alpha$ or $\beta$ but $w_{i} \neq w_{i+1}, i=1, \ldots, k-1$, and $p_{i} \in \mathbb{Z} \backslash\{0\}=\mathbb{Z}^{*}$. Consider $\sigma \in A_{k}$, we will estimate $\rho(0, \sigma(0))$ from below. Let $h$ denote the length of the shortest geodesic in $\Omega$ which surrounds $\bar{\Delta}(1, \epsilon)$. This number $h$ depends on $\epsilon$ and $\eta$ but there exist $h_{0}=h_{0}(\epsilon)$ which depends only on $\epsilon$ so that $h \geqslant h_{0}$. (This could be seen by using the convergence results in $[\mathrm{H}]$ ).

The segment from 0 to $\sigma(0)$ is mapped by $F$ onto a curve $\hat{\sigma}$ which is locally a geodesic and

$$
\rho(0, \sigma(0))=l_{\Omega}(\hat{\sigma}) \quad(=\text { the Poincaré length of } \hat{\sigma}) .
$$

With this information we may estimate $l_{\Omega}(\hat{\sigma})$ from below as follows:

$$
l_{\Omega}(\hat{\sigma}) \geqslant\left(\sum_{j=1}^{k}\left|p_{j}\right|-k\right) h_{0}+k\left(\frac{1}{\eta}\right) .
$$

For the length of a curve connecting the short sides of the tunnel is at least $1 / \eta$ and $\hat{\sigma}$ «contains» $k$ arcs connecting these short sides.

For a vector $v$ in $(\mathbb{N}-\{0\})^{k}$ we write

$$
\|v\|=\sum_{j=1}^{k}\left|v_{j}\right|
$$


Then we have that

$$
\begin{aligned}
\sum_{\gamma \in A_{k}} e^{-s \rho(0, \gamma(0))} & \leqslant 2^{k} \sum_{v \in(\mathbb{N}-\{0\})^{k}} e^{-s\left(\|v\| h_{0}+k\left(1 / \eta-h_{0}\right)\right)} \\
& =2^{k} e^{-s k\left(1 / \eta-h_{0}\right)} \sum_{v \in(\mathbb{N}-\{0\})^{k}} e^{-s h_{0}\|v\|} \\
& =2^{k} e^{-s\left(1 / \eta-h_{0}\right) k}\left[\frac{e^{-s h_{0}}}{1-e^{-s h_{0}}}\right]^{k}
\end{aligned}
$$

and given $s$ if we choose $\eta$ small enough we have that

$$
e^{-s\left(1 / \eta-h_{0}\right)}<\frac{1}{4}\left(e^{s h_{0}}-1\right)
$$

and then the sum (7.2) is majorized by

$$
1+\sum_{k=1}^{\infty} \frac{1}{2^{k}}=2
$$

Remark. The example shows that one can have $\delta$ small while $U_{1}$ is unbounded. On the other hand Theorem 3 shows that for plane domains if $U_{1}$ is bounded then $\delta<1$. This last fact does not hold for Riemann surfaces. Consider a $Z^{3}$-cover $R$ of a compact Riemann surface $S$. Now $R$ has a Green's function (see, e.g., [T, p. 484]) and since $U_{1}$ is invariant under the $Z^{3}$-action we have that $U_{1}$ of $R$ is bounded. On the other hand it is easy to see that the infimum of the Rayleigh's quotient is zero, and so $\delta=1$. This example was pointed out by A. Ancona.

\section{References}

[Ah] Ahlfors, L. Conformal invariants, McGraw Hill, 1973.

[A] Ancona, A. On strong barriers and an inequality of Hardy for domains in $\mathbb{R}^{n}$, J. London Math. Soc. 34(2)(1986), 274-290.

[BP] Beardon, A., Pommerenke, Ch. The Poincaré metric of a plane domain, J. London Math. Soc. 18(2)(1978), 475-483.

[B] Brown, B. Hyperbolic convexity and level sets of analytic functions, Indiana Univ. Math. J. 32(1983), 830-841.

[C] Carleson, L. On $H^{\infty}$ in multiply connected domains. Conference in Harmonic Analysis in honour of Antoni Zygmund, Wadsworth, 1983.

[F] Fernández, J.L. Singularities of inner functions, Math. Z. 193(1986), 393-396.

[FH] Fernández, J. L., Hamilton, D. H. Length of curves under conformal mappings, Comm. Math. Helv. 62(1987), 122-134.

[FHM] Fernández, J. L., Herinonen, J., Martio, O. Quasilines and conformal mappins, Journal d'Analyse Math. 52(1989), 117-132. 
[GGJ] Garnett, J., Gehring, F., Jones, P. Conformal invariant length sums, Indiana Univ. Math. J. 32(1983), 809-829.

[GJ] Garnett, J., Jones, P. The Corona theorem for Denjoy domains, Acta Math. 155(1985), 27-40.

[JM] Jones, P., Marshall, D. Critical points of Green's function, harmonic measure and the Corona problem, Ark. Mat. 23(1985).

[HW] Hayman, W., Wu, J. M. Level sets of univalent functions, Comm. Math. Helv. 56(1981), 366-403.

[H] Hejhal, D. Poincaré metric for variable regions, Math. Z. 137(1974), 7-20.

[Pa1] Patterson, S. J. Spectral theory and Fuchsian groups, Math. Proc. Cambridge Phil. Soc. 81(1977), 59-75.

[Pa2] -, Some examples of Fuchsian groups, Proc. London Math. Soc. 39(3)(1979), 276-298.

[Po1] Pommerenke, Ch. Uniformly perfect sets and the Poincaré metric, Ark. Math. 32(1979), 192-199.

[Po2] -, On uniformly perfect sets and Fuchsian groups, Analysis, 4(1984), 299-321.

[Po3] -, On the Green's fundamental domain, Math. Z. 156(1977), 157-164.

[Po4] -, On Fuchsian groups of accessible type, Ann. Acad. Sci. Fenn. 7(1982), 249-258.

[Po5] - On the Green's function of Fuchsian groups. Ann. Acad. Sci. Fenn. 2(1976), 409-427.

[RR] Rubel, L., Ryff, J. The bounded weak star topology and bounded analytic functions, J. Funct. Anal. 5(1970), 167-183.

[S1] Sullivan, D. Related aspects of positivity in Riemannian Geometry, J. Diff. Geom. 25(1987), 327-351.

[S2] -, The ergodic theory at infinity of a discrete group of hyperbolic motions, Ann. of Math. Studies, 97, 465-496.

[Su] Suzuki, M. Comportement des applications holomorphes autour d'un ensemble polaire, C.R. Acad. Sci. Paris, 304(1987), 191-194.

[T] Tsuji, M. Potential theory in modern function theory, Chelsea, New York, 1970.

[Z] Zinsmeister, M. Espaces de Hardy et domaines de Denjoy, Ark. Math. 27(1989), 363-378.

Recibido: 12 de diciembre de 1988.

José L. Fernández*

Departamento de Matemáticas

Universidad Autónoma de Madrid

28049 Madrid

SPAIN

* Research supported in part by a NSF grant. 